\title{
ESTIMACIÓN DE LA HUMEDAD DEL SUELO A NIVELES DE CAPACIDAD DE CAMPO Y PUNTO DE MARCHITEZ MEDIANTE MODELOS PREDICTIVOS A ESCALAREGIONAL
}

\author{
Pedro Pérez Cutillas \\ Departamento de Geografía. Universidad de Murcia. Centro de Edafología y Biología Aplicada del Segura, \\ Consejo Superior de Investigaciones Cientificas (CEBAS-CSIC) \\ pedrope@um.es, perezcutillas@cebas.csic.es \\ Gonzalo G. Barberá \\ Centro de Edafología y Biología Aplicada del Segura, Consejo Superior de Investigaciones Cientificas (CEBAS-CSIC) \\ gbarbera@cebas.csic.es \\ Carmelo Conesa García \\ Departamento de Geografía. Universidad de Murcia \\ cconesa@um.es
}

\section{RESUMEN}

La estimación de valores edáficos mediante el empleo de técnicas de modelización espacial, basada en procesos y variables ambientales, puede ser una solución válida para solventar la escasez de datos relativos a la información de las propiedades del suelo en estudios aplicados a grandes áreas de trabajo. Para este trabajo se han empleado valores del potencial de succión a niveles de Capacidad de Campo $(p F 2,5)$ y Punto de Marchitez $(p F 4,2)$ obtenidos a partir de datos de 307 perfiles (Proyecto LUCDEME) distribuidos en la Región de Murcia (más de $10000 \mathrm{~km}^{2}$ ), con el fin de proporcionar una estimación del contenido de humedad del suelo a los niveles de $p F$ mencionados mediante 21 variables ambientales utilizadas como predictores. Los resultados aportados muestran claras diferencias en las variables que componen cada uno de los modelos, lo que sugiere que los procesos que determinan los valores de $p F 2,5$ y $p F$ 4,2 en el suelo pueden ser distintos. Entre las variables más

Fecha de recepción: mayo 2013.

Fecha de aceptación: febrero 2014. 
influyentes destacan las asociadas al relieve (i.e. modelo digital de elevaciones, curvaturas), la acumulación de flujo vinculada a las redes de drenaje, y los valores obtenidos mediante técnicas de teledetección, como el NDVI.

Palabras clave: propiedades del suelo, potencial hídrico, modelización, SIG, variables ambientales, teledetección.

\section{ABSTRACT}

Estimating soil values by using spatial modeling techniques based on processes and environmental variables can resolve the scarcity of data concerning from soil properties in studies applied to large areas. In this paper we have used values of soil water potential, on levels for Field Capacity ( $p F 2.5$ ) and Wilting Point ( $p F$ 4.2), obtained from 307 profiles (LUCDEME Project) distributed in the region de Murcia (over $10000 \mathrm{~km}^{2}$ ), for the purpose of provide an estimate of the moisture content of soil $p F$ mentioned levels by 21 environmental variables used as predictors. The supplied results show distinct differences in the variables that compose each of the models, which suggest that the processes that determine the values of $p F 2.5$ and $p F 4.2$ in soil can be different. Among the most influential variables include those associated with topography (digital elevation model, curvatures), flow accumulation linked to drainage networks, and values obtained by remote sensing techniques, such as NDVI.

Keywords: soil properties, water potential, modeling, GIS, environmental variables, remote sensing.

\section{INTRODUCCIÓN}

El contenido de la humedad del suelo es un factor esencial en la dinámica de los flujos hídricos de las regiones semiáridas (Dunkerley, 2002). Por lo general, se produce una disminución de la capacidad de infiltración de los suelos a medida que la humedad se incrementa (Manfreda et al., 2010), situación que se agrava en episodios tormentosos, cuando los volúmenes de precipitación en superficie superan la capacidad de infiltración del suelo llegando al estado de saturación, proceso que se conoce como 'Infiltration excess overland flow' (Herwitz, 1986; Garcia-Estringana et al., 2010). Escorrentía y humedad del suelo se presentan como elementos dependientes en los procesos hidrológicos, siendo ésta última un factor de considerable influencia en la economía hídrica de las plantas (Bucci et al., 2009), entendida como el balance de flujos entre suelo, planta y atmosfera. La vegetación natural, y en menor medida los cultivos (Loussert, 1992; Agustí, 2003), dependen del balance entre el agua que la planta absorbe del suelo y la que pierde por transpiración. Un exceso de transpiración respecto a la absorción genera un balance hídrico negativo con consecuencias negativas para el crecimiento y la producción en los cultivos. Por lo tanto, resulta interesante conocer cómo puede influir la dinámica del agua subsuperficial en la vegetación mediante el estudio de las propiedades hídricas del suelo.

Conocer las relaciones existentes entre potencial matricial $(\psi)$ y el contenido de agua en el suelo $(\theta)$ resulta especialmente útil para describir las propiedades hidrodinámicas del 
suelo. El contenido hídrico se halla normalmente regulado por la capacidad de cada suelo para la retención de agua a una determinada presión negativa, de modo que la disponibilidad del agua vendrá determinada por la relación de estos procesos, junto a la humedad precedente en los sistemas hidrológicos en estudio (Malik et al., 1987). La curva característica de retención de humedad expresa la relación entre el contenido de agua $\left(\theta ; \mathrm{m}^{3} \mathrm{~m}^{-3}\right)$ y el potencial matricial del suelo $(\psi, \mathrm{Pa})$. Esta correspondencia proporciona información sobre características importantes como la capacidad máxima de almacenamiento de agua en el suelo, la capacidad de campo y el punto de marchitamiento. Tales procesos actúan de manera asimétrica por el efecto de histéresis en el estado de capilaridad del agua en el suelo, lo cual origina un comportamiento diferencial entre las fases de absorción de y de desorción producidas en los poros (Richards, 1931), y con ello distintos comportamientos de tensión y deformación dependiendo del grado de saturación en que se encuentra el suelo (Wheeler et al., 2003). La curva de retención de humedad del suelo se ha parametrizado de diversos modos, siendo la formulación de Van Genuchten (1980) la más popular.

La medición de estas propiedades hídricas, tanto en campo como en laboratorio, es compleja en tiempo y recursos, además de demandar un gran número de muestras debido a la variabilidad espacial del suelo (Klute, 1986). Y a pesar que numerosos estudios reconocen la utilidad de los modelos de regresión predictivos para obtener funciones hidráulicas del suelo a partir de propiedades de éste en grandes extensiones (Wosten y Van Genuchten, 1988; Schuh y Cline, 1990; Twarakavi et al., 2010), son, en cambio, escasos los trabajos referidos al análisis y aplicación de técnicas que, basadas en el uso de modelos estadísticos, tratan de estimar las propiedades hidráulicas del suelo a escala regional mediante variables ambientales. Los procesos de cálculo, desarrollados en estos últimos, permiten obtener resultados con una distribución espacial acorde a las características ambientales del entorno analizado, al mismo nivel que otras técnicas de estimación directa generadas a través de métodos de interpolación como cokriging (McBratney et al., 1981), o procesamientos de regresión ponderada geográficamente (GWR) (Wang et al., 2013). Es conocido que las propiedades hidráulicas en el suelo tienen una alta variabilidad espacial, y están condicionadas por la combinación de numerosos factores ambientales (Poggio et al., 2010; Bandaraet al., 2013).

Estas técnicas que están basadas fundamentalmente en la dependencia espacial de las variables de estudio, está señalada para áreas no muy grandes y con muestreos bastante intensivos y regulares (Chen et al. 2000; Fox and Sabbagh 2002). En una estimación de carácter regional (entendida como un área extensa de miles de kilómetros cuadrados), con muestras ampliamente dispersas, la información proporcionada por la posible autocorrelación espacial entre muestras puede ser muy reducida (Burrough et al., 1997; Westernet al., 2004).Como la densidad de medidas no es habitualmente muy alta a escala regional, se requiere el uso de otros métodos para predecir los valores de las variables del suelo de manera sencilla y asequible (Ließ et al., 2012). Una alternativa particularmente efectiva es la modelización de la relación entre la variable de interés en el suelo y las variables ambientales de las que se dispone de información espacialmente distribuida (Gessler et al., 1995; Brus y De Gruijter, 1997; McKenzie y Ryan, 1999; Thompson et al., 2001; McBratney et al., 2003). La importancia de dicha relación en el proceso de elaboración de la cartografía digital de suelos ha sido ya reseñada por diversos autores mediante el uso de métodos estadísticos aplicados a la predicción espacial de las propiedades del suelo (Minasny et al., 2008; Dobos 
and Hengl, 2009; Bou Kheir et al., 2010; Greve et al., 2012). El resultado son productos cartográficos con información continua obtenidos mediante modelos estadísticos de las propiedades del suelo en función de las variables ambientales que influyen en su formación y distribución espacial.

Desde otra perspectiva, la teledetección va a permitir una visión general de grandes superficies, proporcionando volúmenes masivos de datos cuantitativos georreferenciados, y valores espectrales que presentan buenas correlaciones con las coberturas de los usos del suelo, además de poseer una capacidad de actualización temporal muy dinámica(BenDor, 2002). La evaluación, caracterización y determinación de las propiedades del suelo, mediante la utilización de datos procedentes de sensores remotos ha sido extensamente aplicada durante los últimos años (Scull et al., 2002; Rawls et al., 2004; Uno et al., 2005; Vrieling, 2006; Lagacherie et al., 2012; Poggio et al., 2013). La capacidad de la espectrometría en condiciones de laboratorio está demostrada para lapredicción de importantes propiedades del suelo (Schulten y Schnitzer, 1997; Viscarra Rossel et al., 2006; Ben-Dor et al., 2009). Sin embargo, su aplicación directa a la superficie terrestre no está exenta de ciertas dificultades y limitaciones, lo que hace aconsejable, en este caso, enfocar su uso a la obtención de índices específicos relacionados con las variables ambientales

Los resultados de los trabajos mencionados han servido para plantear aquí procesos de modelización, mediante análisis de regresión con una serie de variables ambientales, necesarios para el cálculo de los valores de capacidad de campo y del punto de marchitamiento de la curva de retención de humedad del suelo.

\section{II. ÁREA DE ESTUDIO Y CONDICIONES AMBIENTALES}

Como área de estudio se ha elegido la Región de Murcia (11313 km², INE, 2013), encuadrada dentro de la cuenca del Río Segura $\left(18208 \mathrm{~km}^{2}\right.$, CHS, 2013) en el Sureste de España (Fig. 1). En conjunto, se trata de una superficie bastante accidentada por la presencia de numerosas sierras, pertenecientes a las Cordilleras Béticas, que se alinean en dirección ENEWSW con altitudes a menudo superiores a los $1.000 \mathrm{~m}$. Entre ellas se intercalan una serie de valles, cubetas, llanuras y altiplanos, que en conjunto configuran una topografía contrastada y un territorio singular de gran variedad paisajística. La zona de estudio participa en su mayor parte de las características de un clima mediterráneo semiárido (Capel-Molina, 2000): precipitación media de la cuenca en torno a $375 \mathrm{~mm} /$ año, con valores medios anuales de 472 mm en cabecera y de $317 \mathrm{~mm}$ en zonas próximas a la desembocadura, gran irregularidad pluviométrica estacional e interanual, sequías prolongadas y lluvias a menudo torrenciales.

Toda esta región recibe una fuerte insolación anual, con un promedio de 2800 horas de sol al año, un máximo en julio (340 h) y un mínimo en diciembre (160 h), según datos del observatorio de Alcantarilla, próximo a la ciudad de Murcia (Alonso Sarria, 2007). Por el carácter accidentado del relieve regional, las variaciones locales en la radiación recibida son notables. Sólo en las tierras más llanas (llanura litoral, depresiones pre-litorales y altiplanicies) los contrastes disminuyen y la homogeneidad es mayor. Estimaciones basadas en modelos teóricos de terreno han dado valores medios de $5.023 \mathrm{kWh} / \mathrm{m}^{2} / \mathrm{año}$, valores que superan los 5.500 e incluso los 6.000 en solana y descienden en la umbría, especialmente en las zonas de mayor altitud (Conesa García y Alonso Sarría, 2006). 


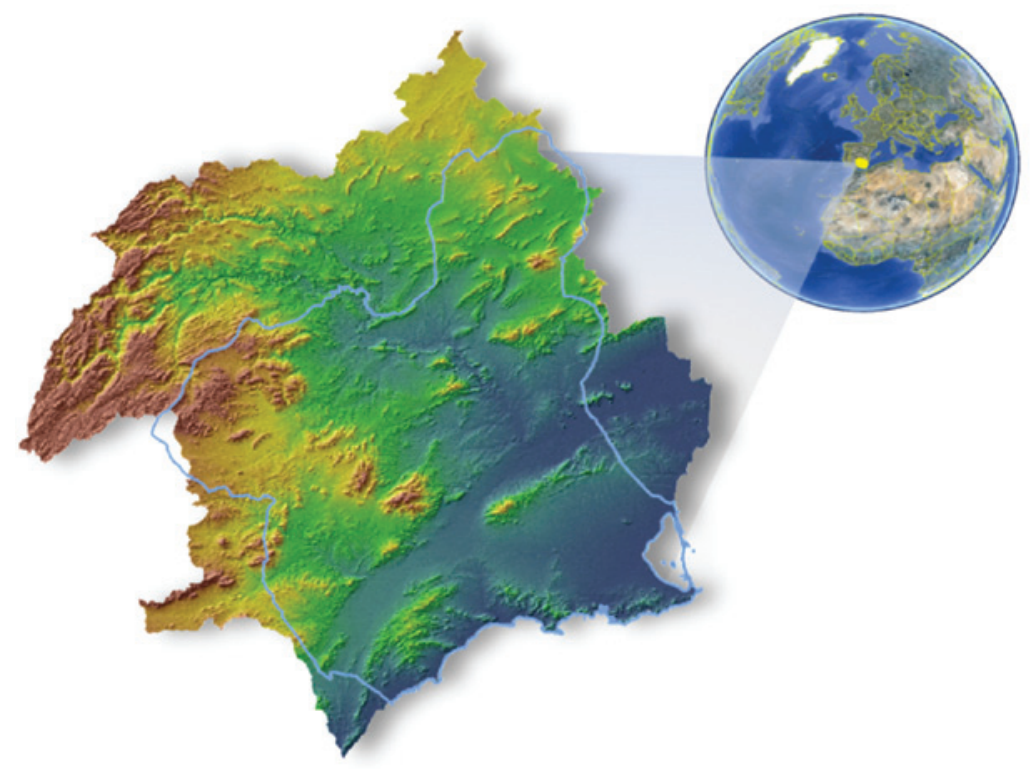

La desertificación y la pérdida de suelo por erosión se presentan como unas de las causas más importantes de la degradación del suelo en este territorio, ya que implica una pérdida de sus principales componentes físicos, químicos y biológicos (Boix-Fayos et al., 2005). Según datos del Inventario Nacional de Erosión de Suelos, elaborado por el Ministerio de Medio Ambiente (MMA, 2002-2012), la erosión media del suelo en la Cuenca del río Segura se sitúa en torno a la media española, 24,53 ton $/ \mathrm{ha}^{-1} \mathrm{año}^{-1}$, equivalentes a un rebajamiento de casi $2 \mathrm{~mm}$ al año. En esta cuenca existe una gran diversidad de suelos originados por la acción de diferentes procesos asociados a la influencia de múltiples factores ambientales. Entre ellos destacan las condiciones climáticas, la litología y el relieve, que condicionan en gran medida la erosión y los procesos edáficos (Álvarez Rogel et al., 2001).

\section{MATERIAL Y MÉTODOS}

\section{III.1. Datos analíticos del Proyecto LUCDEME}

Toda la información empírica sobre el potencial de succión $(p F)$ en el suelo ha sido obtenida a partir de datos del Proyecto de Lucha contra la Desertificación en el Mediterráneo (LUCDEME) (ICONA, 1986), elaborado a comienzos de los años ochenta y primeros de los noventa. La información utilizada en esta parte del trabajo se centra en los datos completos de una selección de perfiles edáficos, muestreados en el campo y analizados en el laboratorio. Las diferentes unidades taxonómicas del sistema FAO-UNESCO (1974), extraídas del 
proyecto LUCDEME para la zona de estudio, están caracterizadas por 307 perfiles, muestreados ad-hoc, distribuidos por el territorio en los lugares donde mejor representadas se hallan dichas unidades. La información de los perfiles aparece clasificada por profundidades de los diferentes horizontes observados en campo, en función de sus propiedades físicoquímicas anotadas en 1880 registros.

El empleo de variables ambientales para la estimación de los valores de contenido de humedad a escala regional aconseja filtrar estos muestreos a los niveles superiores del perfil para una mejor conexión con la descripción de la información ambiental. De las determinaciones analíticas de los perfiles se han suprimido los horizontes orgánicos (parte externa del suelo) y los horizontes más profundos (Fig. 2).

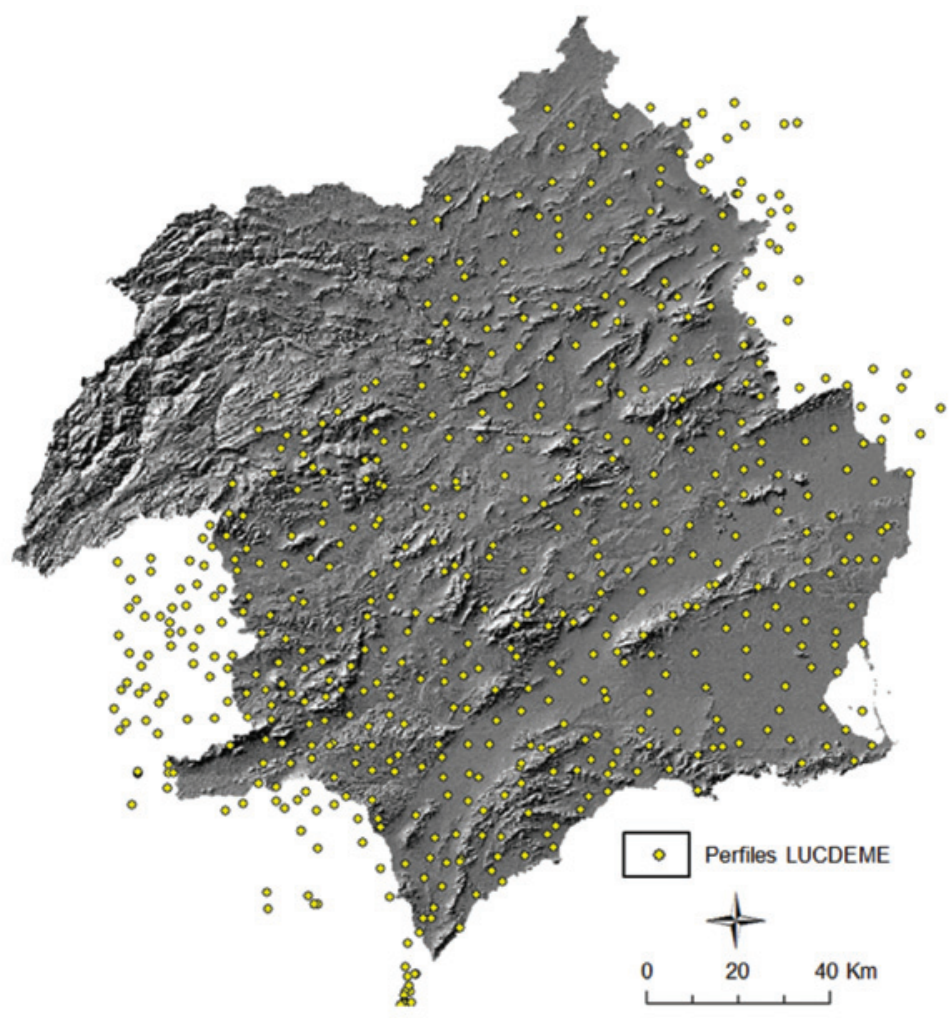

\section{III.2. Variables ambientales empleadas en la modelización}

En sentido estricto no se pueden denominar variables ambientales de obtención directa a todas las variables utilizadas en los procesos de modelización de este estudio. Algunas de 
ellas son índices elaborados a partir de señales espectrales de sensores remotos que sólo se pueden relacionar indirectamente con las características del suelo. No obstante, mantenemos esta denominación para homogeneizar toda la información tratada en los análisis de SIG y tratamientos estadísticos. Estas variables se pueden estructurar en una serie de conjuntos de datos, que son descritos en los siguientes bloques de variables (entre paréntesis y en negrita figura el número de secuencia de cada variable):

\section{III.2.1. Variables topográficas e hidrológicas}

Estas variables se han obtenido a partir del Modelo Digital del Terreno (DTM) de la misión TERRA de la NASA, realizado con el sensor japonés ASTER (NASA - METI, 2013). A partir de este DTM (1) se ha obtenido: Pendientes (2), utilizando el método de cambio máximo en el valor de altitud respecto de los píxeles circundantes para cada píxel, con valores expresados en grados de inclinación (Burrough and McDonnell, 1998). La curvatura que se calcula de manera análoga al mapa de pendientes, pero mediante la segunda derivada, expresándose como la pendiente de la pendiente (Moore et al., 1991). La curvatura se puede descomponer (Zeverbergen and Thorne, 1987) en Curvatura del perfil (3), que se calcula a partir de la dirección de máxima pendiente, y curvatura perpendicular (4).Y la acumulación de flujo (5) que representa el número de píxeles de la cuenca vertiente a un píxel en particular (Tarboton et al., 1991). Indicar que la distribución de esta variable tiene una distribución fuertemente sesgada a la derecha por lo que para la modelización se ha utilizado su logaritmo.

\section{III.2.2. Tipos de suelos y litología}

La información edafológica y litológica procede de diferentes fuentes. Mapa de suelos del proyecto LUCDEME (E. 1:100.000) (6) con la distribución espacial de las unidades taxonómicas clasificadas según el Sistema FAO 1974, la mayoría de los polígonos del suelo no identifican una unidad taxonómica sino una asociación de dos o tres unidades. Además el mapa de suelos cartografía la Fase Salina (7), identificada como áreas cuya conductividad eléctrica $>2 \mathrm{mmhos} / \mathrm{cm}$ Mapa de litología (8) realizado a partir de los datos del Mapa Geológico Nacional 1:50.000 (MAGNA) del Instituto Geológico y Minero de España (IGME), cartografía geológica que ha sido reclasificada para obtener los valores litológicos, mediante interpretación de la leyenda de cada formación geológica que aparece en la memoria que acompaña a cada hoja del mapa

\section{III.2.3. Variables climáticas}

Las variables climáticas utilizadas son la capa de precipitación $(\mathrm{mm})(\mathbf{9})$ y la de temperatura $\left({ }^{\circ} \mathrm{C}\right)(\mathbf{1 0})$ para la zona de estudio, elaboradas por el Laboratorio de Biogeografía Informática (LBI, 2013) del Museo Nacional de Ciencias Naturales del CSIC y diversas variables relacionadas con la radiación solar.

La radiación solar recibida por un punto en un momento depende de la posición del sol respecto a la superficie receptora que a su vez está determinado por la latitud y el momento 
del año. La cantidad de radiación solar que recibe una superficie es sólo una porción de lo que recibiría fuera de la atmósfera debido a la transmisividad de esta, influyendo solo la proporción de radiación solar capaz de atravesarla (Rich et al., 1994). El cálculo de la radiación se ha realizado para un año completo, obteniéndose tres capas de información: La radiación solar directa (11)(Fu and Rich, 2000), es decir, la radiación que la superficie recibe directamente del sol, (12) la radiación solar difusa (Fu and Rich, 2000), correspondiente a la radiación que alcanza un punto no directamente del sol sino de la proporción que ha sido dispersada por la atmósfera. Finalmente se incluyó una última capa que representa la duración de la radiación solar directa (13), indicada en horas de insolación directa para cada uno de los píxeles.

\section{III.2.4. Variables complementarias obtenidas mediante Teledetección}

Se han utilizado imágenes del satélite Landsat 5 para obtener información adicional, mediante el uso de dos fechas (14/02/2009 y 24/07/2009). Estas imágenes corresponden a dos periodos estacionales diferentes del mismo año, uno estival y otro invernal. De esta forma se minimiza el posible sesgo estacional. Para cada periodo se estimó el Índice de Vegetación de Diferencia Normalizada (NDVI) (14 y 15, datos para invierno y verano, respectivamente). Este índice permite determinar la cantidad, calidad y desarrollo de la vegetación de acuerdo con la medición de la intensidad de la radiación de las longitudes de onda de la zona Roja (R) e Infrarrojo Cercano (IRC) del espectro electromagnético que la vegetación emite o refleja (Townshend et al, 1985). Este índice aprovecha la diferencia de las características espectrales de estas dos bandas, donde las altas absorciones de pigmentos de clorofila en la banda Roja contrastan con la alta reflectividad de los materiales vegetales de la banda IRC.

$$
\mathrm{NDVI}=(\mathrm{IRC}-\mathrm{R}) /(\mathrm{IRC}+\mathrm{R})
$$

Donde $I R C$ es el valor de los píxeles en la banda del Infrarrojo Cercano $(0,75-0,90 \mu \mathrm{m})$ y $R$ el valor de los píxeles en la banda Roja $(0,63-0,69 \mu \mathrm{m})$.

También se han aplicado una serie de algoritmos sobre las imágenes seleccionadas para obtener indicadores mineralógicos (Sabins, 1981; Crosta et al., 2003), mediante combinación de las siguientes funciones estandarizadas «Clay Minerals» (minerales de arcilla, CMI) (16 y 19), «Ferrous Minerals» (minerales ferrosos, FMI) (17 y 20) y «Iron Oxide» (óxidos de hierro, IOI) (18 y 21). Para cada una de las funciones se han realizado los cálculos correspondientes a la estación de verano (Julio) e invierno (Febrero).

El primer índice (CMI) se deriva de la alta reflectancia de los minerales arcillosos al

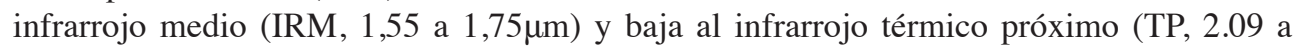
$2,35 \mu \mathrm{m})$, mientras que una roca no alterada tiene una reflectancia relativamente uniforme en las dos longitudes de onda. El índice FMI se ha empleado para realzar áreas con alto contenido ferroso (Dury, 1987), aplicando el mismo principio que en el caso anterior, en concreto el cociente entre el intervalo de longitud de onda del infrarrojo medio (IRM, 1,55 a 1,75 $\mu \mathrm{m}$ ), donde estos minerales ofrecen una gran reflectividad, y los del infrarrojo cercano (IRC, 0,75-0,90 $\mu \mathrm{m})$, con elevados niveles de absorción. Los minerales con contenido en óxido de hierro tienen valores elevados de reflectancia correspondientes al intervalo de longitud de 
ondas de la banda Roja (R, 0,63-0,69 $\mu \mathrm{m})$ y valores más bajos de reflectancia en el intervalo de longitud de la banda Azul (A,0,45-0,515 $\mu \mathrm{m})$, mientras que una roca sin minerales de este tipo registra valores semejantes en las bandas roja y azul. Mediante el índice IOI que resulta del cociente de valores entre ambas bandas, pueden distinguirse rocas formadas por minerales con cierto contenido en hierro, posiblemente afectadas por procesos de alteración hidrotermal. En el cálculo de dichos índices se han empleado los siguientes algoritmos:

\section{Índice de Minerales Arcillosos (CMI) $=$ IRM / TP \\ Índice de Minerales Férreos $(\mathrm{FMI})=\mathrm{IRM} / \mathrm{IRC}$ \\ Índice de Óxido de Hierro $(\mathrm{IOI})=\mathrm{R} / \mathrm{A}$}

Donde $A$ es el valor de los píxeles en la banda Azul $(0,45-0,515 \mu \mathrm{m}) ; R$, el valor de los píxeles en la banda Roja $(0,63-0,69 \mu \mathrm{m})$; $I R C$, el valor de los píxeles en la banda del Infrarrojo Cercano $(0,75-0,90 \mu \mathrm{m})$; IRM, el valor de los píxeles en la banda del Infrarrojo Medio (1,55-1,75 $\mu \mathrm{m}) ; T P$, el valor de los píxeles en la banda del Térmico Próximo (2,09-2,35 $\mu \mathrm{m})$.

A continuación (Tabla 1) se exponen los valores resumen de cada una de las variables expuestas:

Tabla 1

DESCRIPCIÓN DE LA REPRESENTATIVIDAD DE LOS VALORES DE LAS VARIABLES AMBIENTALES RASTER Y SU ESTADÍSTICO DESCRIPTIVO CON EL CÁLCULO DEL RANGO, MEDIA Y DESVIACIÓN ESTÁNDAR

\begin{tabular}{|l|l|l|l|c|c|c|}
\hline Id & Variable & Descripción valores mínimos & Descripción valores máximos & Rango & Media & $\begin{array}{c}\text { Desv. } \\
\text { Estándar }\end{array}$ \\
\hline $\mathbf{( 1 )}$ & DTM & Altitud nivel mar & Altitud absoluta & $0-2057$ & 612 & \pm 407 \\
\hline $\mathbf{( 2 )}$ & Slp & Superficies llanas & Relieve escarpado & $0-79$ & 7,5 & $\pm 7,7$ \\
\hline $\mathbf{( 3 )}$ & $\mathbf{C u P}$ & Curvatura Cóncava & Curvatura Convexa & $-1,6$ & 0,008 & $\pm 0,27$ \\
\hline $\mathbf{( 4 )}$ & $\mathbf{C u P P}$ & Curvatura Convexa & Curvatura Cóncava & -6 & 0,008 & $\pm 0,41$ \\
\hline $\mathbf{( 5 )}$ & FAc & Baja Acumulación de flujo & Alta Acumulación de flujo & $0-17$ & 1,6 & $\pm 1,8$ \\
\hline $\mathbf{( 9 )}$ & $\mathbf{P r}$ & Precipitación mínima & Precipitación máxima & $250-771$ & 390 & \pm 84 \\
\hline $\mathbf{( 1 0 )}$ & Tmp & Bajas temperaturas & Altas temperaturas & $8,2-18,4$ & 15,1 & $\pm 2,07$ \\
\hline $\mathbf{( 1 1 )}$ & RaDir & Baja radiación directa & Alta radiación directa & $0,001-2,258 *$ & $1,36 *$ & $\pm 0,12$ \\
\hline $\mathbf{( 1 2 )}$ & RaDif & Baja radiación difusa & Alta radiación difusa & $0,083-0,514 *$ & $0,28 *$ & $\pm 0,01$ \\
\hline $\mathbf{( 1 3 )}$ & RaDur & Escasa duración de radiación & Elevada duración de radiación & $40-4360$ & 4,03 & $\pm 0,27$ \\
\hline $\mathbf{( 1 4 )}$ & NDVIfeb & Suelos artificiales - agua & Abundante vegetación & -2 & 0,05 & $\pm 0,19$ \\
\hline $\mathbf{( 1 5 )}$ & NDVIjul & Suelos artificiales - agua & Abundante vegetación & -2 & 0,03 & $\pm 0,13$ \\
\hline $\mathbf{( 1 6 )}$ & CMIfeb & Baja alteración minerales CMI & Alta alteración minerales CMI & $0-3,5$ & 1,21 & $\pm 0,97$ \\
\hline $\mathbf{( 1 7 )}$ & FMIfeb & Baja alteración minerales FMI & Alta alteración minerales FMI & $0-3$ & 0,83 & $\pm 0,75$ \\
\hline $\mathbf{( 1 8 )}$ & IOIfeb & Baja alteración minerales IOI & Alta alteración minerales IOI & $0-1,5$ & 0,31 & $\pm 0,28$ \\
\hline $\mathbf{( 1 9 )}$ & CMIjul & Baja alteración minerales CMI & Alta alteración minerales CMI & $0-3,5$ & 1,21 & $\pm 0,92$ \\
\hline $\mathbf{( 2 0 )}$ & FMIjul & Baja alteración minerales FMI & Alta alteración minerales FMI & $0-3$ & 0,95 & $\pm 0,76$ \\
\hline $\mathbf{( 2 1 )}$ & IOIjul & Baja alteración minerales IOI & Alta alteración minerales IOI & $0-1,5$ & 0,39 & $\pm 0,34$ \\
\hline * RaDr y RaDi están escalados a 1/1000000 respecto a los valores originales. En la tabla están expresados en MWh/m². & \\
\hline
\end{tabular}




\section{III.3. Modelos predictivos de estimación del contenido de humedad para los valores de capaci- dad de campo y punto de marchitez}

Los procedimientos SIG se implementaron mediante ArcGIS v.10 (ESRI, 2013). A partir de las coordenadas de las muestras de la base de datos se obtuvieron los valores de las 21 variables ambientales para píxeles de $400 \mathrm{~m}$ de resolución. Debido a la naturaleza discreta de las variables del bloque SL en los modelos propuestos existe un coeficiente para el efecto de cada nivel de la clase, esto es para cada tipo desuelo o litología. Por razones matemáticas, los niveles de una variable discreta se introducen en un modelo de regresión como un conjunto de variables dummy. Así, se crean tantas variables nuevas cómo niveles tiene la clase, asignando el valor 1 a la variable que representa un nivel particular cuando la muestra en particular pertenece a ese nivel. Con ese sistema decodificación y con la estructura de un modelo de regresión, es preciso que exista un nivel de 'referencia', no necesariamente explícito en la formulación del modelo, en tanto que los coeficientes para el resto de las variables dummy expresan el efecto respecto a esa clase de referencia.

Los valores de $p F$ disponibles para cada punto fueron relacionados en una tabla con los correspondientes a las variables ambientales seleccionadas. Dicha tabla se utilizó como input para el lenguaje de análisis estadístico 'R' (R Core Team, 2013), con el que se construyeron modelos de regresión según el procedimiento descrito más adelante. Un modelo de regresión en el que los predictores son capas de SIG puede representarse fácilmente mediante álgebra de mapas ya que es una simple combinación lineal de dichas capas.

La resolución de pixel para los modelos fue de $400 \mathrm{~m}$, por tratarse de una resolución espacial con cierto detalle y ser acorde con la modelización de estos valores a escala regional. Además, Pérez-Cutillas (2013) mostró que el uso de resoluciones inferiores no mejora de manera sustancial la capacidad predictiva de los modelos. Para uniformizar dicha resolución, se realizó el cambio de tamaño de los píxeles originales para todas las capas utilizadas como variables predictoras en los modelos de $p F$. Para las variables originalmente ráster se remuestreó por interpolación bilineal (Kidner et al., 1999), salvo en el caso de las variables climáticas de temperatura y precipitación, que se han introducido en los modelos en una sola resolución con su tamaño original de $850 \mathrm{~m}$. En el caso de las capas vectoriales los polígonos fueron remuestreados a ráster con una resolución de $400 \mathrm{~m}$, de tal forma que un píxel es siempre asignado al polígono que cubre mayor superficie dentro del mismo. En la Figura 3 se esquematiza el proceso completo. Los datos analíticos del potencial de succión corresponden a los 307 perfiles de muestreo del proyecto LUCDEME en la zona de estudio.

Para obtener el modelo de $p F$ en función de las variables ambientales se aplicó la regresión lineal paso a paso hacia adelante (forward stepwise) utilizando el Criterio de Información de Akaike (AIC). El AIC fue desarrollado por Hirotsugu Akaike (1974). El método ofrece una medida relativa de la pérdida de información cuando un determinado modelo es utilizado para describir la realidad, de modo que finalmente permite seleccionar el modelo que más se ajusta a los datos reales. Matemáticamente se puede demostrar que AIC mide la distancia entre el modelo ajustado y el modelo perfecto que representa exactamente la realidad, incluso aunque este modelo perfecto no nos sea nunca conocido. De esta forma, entre dos modelos ajustados a los mismos datos es preferible aquél cuyo valor de AIC es menor porque está más cercano al modelo perfecto o ideal. Hay 
que señalar que un pequeño descenso en los valores calculados en el AIC representa una mejora importante en lacalidad del modelo. El AIC se expresa de la siguiente manera:

$$
A I C=2 k-2 \ln (L)
$$

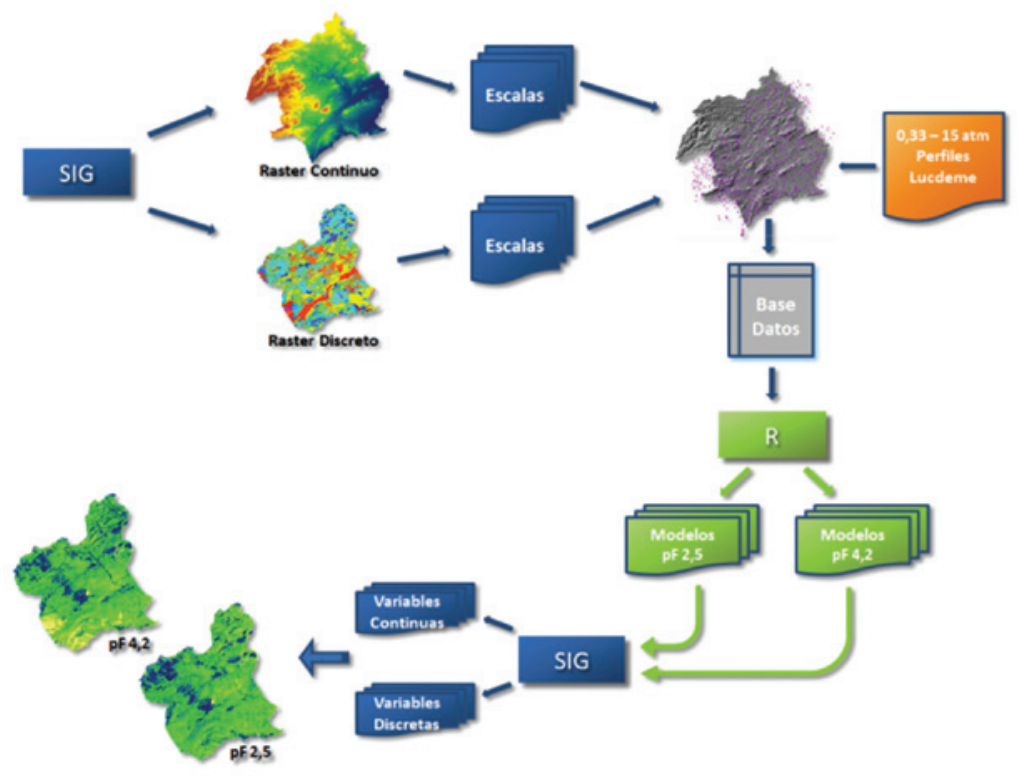

Donde $k$ es el número de parámetros en el modelo, y $L$, el valor máximo de la función de verosimilitud para el modelo estimado. Aplicado a un proceso de selección de modelos paso a paso, de manera resumida, la mecánica es la siguiente. En primer lugar se ajustan modelos con una sola variable, seleccionando entre todos ellos el modelo con el AIC más bajo, y a partir de él se construye una batería de modelos de dos variables. De estas dos variables una es fija, que se seleccionó en el primer paso, mientras que la segunda proviene del subconjunto formado por el resto de las variables. Es decir, se construyen todos los modelos de 2 variables que incluyan como variable la seleccionada en el primer paso. De entre todos ellos se selecciona como mejor modelo aquél con AIC más bajo. Se sigue sucesivamente el proceso con 3, 4, etc. variables hasta que el AIC de un modelo con $n+l$ variables es superior al modelo con $n$ variables. En este sentido el proceso de selección de variables es el clásico de paso a paso hacia delante (forward stepwise), pero el criterio para añadir variables está basado en AIC.

Como la respuesta frente a las variables cuantitativas puede ser no lineal, para todas ellas se utilizó en los modelos términos cuadráticos. Por ejemplo, para la precipitación no se incluyó en la construcción de los modelos sólo la variable precipitación sino también su cuadrática precipitación ${ }^{2}$. De esta forma el modelo puede ajustar fácilmente diversas formas curvas. 


\section{RESULTADOS Y DISCUSIÓN}

\section{IV.1. Modelización de los valores de pF mediante variables ambientales a escala regional}

El mejor modelo predictivo para el contenido de agua en el suelo a valores de capacidad de campo $(p F 2,5)$ generado por el procedimiento descrito y de acuerdo con los valores de AIC obtenidos para cada combinación de variables, se describe en la siguiente formula (los acrónimos de los modelos se describen en la Tabla 1):

$$
\text { Modelo pF 2,5 }=\left[D T M+F A c^{2}+R a D i+R a D r+R a D u^{2}+F M I_{f e b}+N D V I_{j u l}+F S L u\right]
$$

A continuación se muestra el mejor modelo obtenido para los valores del punto de marchitez $(p F 4,2)$ :

$$
\begin{aligned}
\text { Modelo pF 4,2 }= & {\left[D T M+D T M^{2}+C u P+C u P^{2}+F A c^{2}+T m p^{2}+P^{2}+R a D i+R a D r+\right.} \\
& \left.N D V I_{j u l}+L M+F S L u\right]
\end{aligned}
$$

Los modelos obtenidos muestran unos coeficiente de determinación dispares, $\mathrm{R}^{2}=0,15$ para el $p F 2,5$ y $\mathrm{R}^{2}=0,31$ para el $p F 4,2$. Los coeficientes y sus errores estándar calculados para cada una de las variables cuantitativas se muestran en la Tabla 2, mientras que los coeficientes para los diferentes niveles de las variables discreta aparecen indicados en la Tabla 3.

\begin{tabular}{|c|c|c|c|c|}
\hline \multirow[b]{2}{*}{ Variable } & \multicolumn{2}{|c|}{$p F 2,5$} & \multicolumn{2}{|c|}{$p F 4,2$} \\
\hline & Estimate & Std. Error & Estimate & Std.Error \\
\hline (Intercept) & $2,96 \mathrm{E}+05$ & $1,02 \mathrm{E}+05$ & $1,08 \mathrm{E}+05$ & $7,06 \mathrm{E}+04$ \\
\hline DTM & $3,69 \mathrm{E}+01$ & $1,21 \mathrm{E}+01$ & $4,45 \mathrm{E}+01$ & $1,60 \mathrm{E}+01$ \\
\hline DTM $^{2}$ & --- & --- & $-1,72 \mathrm{E}-02$ & $6,24 \mathrm{E}-03$ \\
\hline $\mathrm{CuP}$ & --- & --- & $4,85 \mathrm{E}+04$ & $1,74 \mathrm{E}+04$ \\
\hline $\mathrm{CuP}^{2}$ & --- & --- & $-2,40 \mathrm{E}+05$ & $1,24 \mathrm{E}+05$ \\
\hline FAc $^{2}$ & $8,06 \mathrm{E}+01$ & $4,23 \mathrm{E}+01$ & $6,96 \mathrm{E}+01$ & $3,37 \mathrm{E}+01$ \\
\hline $\mathbf{T m p}^{2}$ & --- & --- & $1,46 \mathrm{E}+02$ & $7,53 \mathrm{E}+01$ \\
\hline $\mathbf{P r}^{2}$ & --- & --- & $1,18 \mathrm{E}-01$ & $3,94 \mathrm{E}-02$ \\
\hline RaDi & $-1,16 \mathrm{E}+06$ & $4,14 \mathrm{E}+05$ & $-6,26 \mathrm{E}+05$ & $2,66 \mathrm{E}+05$ \\
\hline RaDr & $1,86 \mathrm{E}+04$ & $9,82 \mathrm{E}+03$ & $1,15 \mathrm{E}+04$ & $7,84 \mathrm{E}+03$ \\
\hline $\mathbf{R a D u}^{2}$ & $1,10 \mathrm{E}+03$ & $7,43 \mathrm{E}+02$ & --- & --- \\
\hline FMIfeb & $-3,21 \mathrm{E}+03$ & $1,18 \mathrm{E}+03$ & --- & --- \\
\hline NDVI $_{\text {Jul }}$ & $1,37 \mathrm{E}+04$ & $4,75 \mathrm{E}+03$ & $1,22 \mathrm{E}+04$ & $3,72 \mathrm{E}+03$ \\
\hline SPLu & $3,43 \mathrm{E}+03$ & $1,40 \mathrm{E}+03$ & $2,30 \mathrm{E}+03$ & $1,09 \mathrm{E}+03$ \\
\hline
\end{tabular}

Tabla 2

VARIABLES Y COEFICIENTES OBTENIDOS EN LA MODELIZACIÓN DE LOS pF 2,5 Y pF 4,2. 
Tabla 3

COEFICIENTES OBTENIDOS EN EL PROCESO DE MODELIZACIÓN DE LOS NIVELES DE pF 2,5 Y pF 4,2 PARA LAS DIFERENTES CATEGORIAS DE LA VARIABLE DISCRETA 'LITOLOGÍAMAGNA' (LM). RELACIÓN DE CLASES Y CÓDIGOS POR MUESTRA: ARCILLAS = 12; ARENISCAS= 14; CALIZAS = 16; COLUVIOS = 17; CONGLOMERADO= 18; CUATERNARIO INDIFERENCIADO= 19; DOLOMÍAS= 20; MARGAS= 23; KEUPER= 25; METAMÓRFICO-SILÍCEO $=26$

\begin{tabular}{|l|c|c|}
\cline { 2 - 3 } \multicolumn{1}{c|}{} & \multicolumn{2}{c|}{$\boldsymbol{p F} \mathbf{4 , 2}$} \\
\hline Variable & Estimate & Std.Error \\
\hline LM_12 & $-2,54 \mathrm{E}+03$ & $2,09 \mathrm{E}+03$ \\
\hline LM_16 & $-1,05 \mathrm{E}+03$ & $2,89 \mathrm{E}+03$ \\
\hline LM_17 & $-2,74 \mathrm{E}+03$ & $1,80 \mathrm{E}+03$ \\
\hline LM_18 & $-4,28 \mathrm{E}+03$ & $1,75 \mathrm{E}+03$ \\
\hline LM_19 & $-3,32 \mathrm{E}+03$ & $1,97 \mathrm{E}+03$ \\
\hline LM_20 & $-3,05 \mathrm{E}+03$ & $1,48 \mathrm{E}+03$ \\
\hline LM_23 & $2,21 \mathrm{E}+03$ & $2,61 \mathrm{E}+03$ \\
\hline LM_25 & $-1,10 \mathrm{E}+03$ & $1,58 \mathrm{E}+03$ \\
\hline LM_26 & $-1,13 \mathrm{E}+03$ & $2,65 \mathrm{E}+03$ \\
\hline
\end{tabular}

La Figura 4 muestra la representación espacial para la estimación del contenido de agua en el suelo a valores de $p F$ 2,5 y pF 4,2 con las variables y coeficientes obtenidos en el modelo.

Figura 4

CAPAS RASTER DE LA ESTIMACIÓN DEL POTENCIAL DE SUCCIÓN (pF 2,5 Y pF 4,2) PARA LA RESOLUCIÓN 400 M (PÍXEL) ELABORADA MEDIANTE VARIABLES AMBIENTALES. EXPRESADA EN \% DEL CONTENIDO GRAVIMÉTRICO DE AGUA EN EL SUELO, EN TONOS OSCUROS LOS VALORES MÁS ALTOS Y EN TONOS CLARO LOS MÁS BAJOS

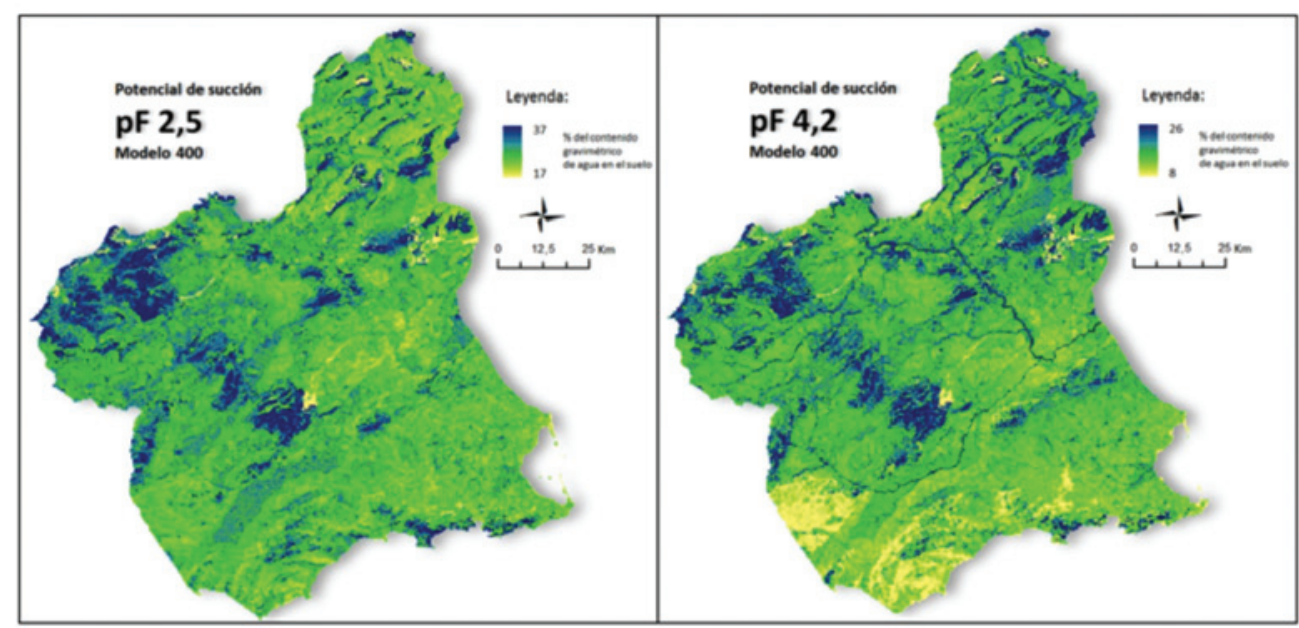




\section{IV.2. Influencia y efecto de las variables ambientales en los modelos de pF}

Para conocer cuál es el efecto que ejercen las distintas variables en los modelos de estimación de los valores de $p F$ se puede obtener una visión de conjunto observando los coeficientes del modelo (Tablas 2 y 3). Los efectos analizados se basan en las repercusiones que producen las variables sobre los distintos modelos a partir de los coeficientes obtenidos en los procesos de modelización. Un signo positivo (+) en estos valores significa que el efecto de la variable aumenta el agua retenida por el suelo a determinado $p F$ mientras que los signos (-) producen el efecto contrario. No obstante esta simple regla puede no ser válida para aquellas variables que entran en el modelo en forma cuadrática, ya que, por denotar curvatura que puede incluir máximos o mínimos que deben ser representadas gráficamente. Esta representación ayuda a comprender cuál es la respuesta, a cambios en las variables ambientales, del contenido de agua del suelo a una succión determinada.

Antes de analizar el efecto de las variables, merece reseñar las desigualdades encontradas entre los dos modelos. De hecho, resultan importantes las diferencias apreciadas respecto al número de variables que componen los modelos, en particular el modelo a $p F 4,2$, más complejo y con mayor predictivo que el modelo a $p F 2,5$. La mayor incertidumbre del modelo que describe la Capacidad de Campo puede deberse a la menor precisión que ofrecen los procesos de medida a menor tensión (Richards, 1947).

La discusión del efecto de las variables sobre los $p F$ es enormemente interesante por las conclusiones que se pueden extraer de las distintas implicaciones que ejercen los patrones ecológicos en su distribución y variabilidad espacial. En la realización de este análisis se ha agrupado las variables en los bloques descritos en la metodología, adoptando igualmente un tratamiento diferente individualizado para la información del bloque SL, por su condición cualitativa. Las variables del bloque TG que aparecen en el modelo $p F$ 2,5 son $[\mathrm{DTM}]$ y $[\mathrm{FAc}]$. En el primer caso observamos cómo el aumento de la altitud aparece relacionado con un crecimiento de los valores de humedad del suelo a Capacidad de Campo. [FAc] se presenta con valores cuadráticos, con un incremento progresivo en los valores estimados de humedad del suelo a $p F$ 2,5 en las áreas con altos valores de acumulación de flujo (Fig. 5). Para el modelo de $p F 4,2$ se repiten los efectos de manera similar para estas variables, con la diferencia de [DTM] se encuentra en el modelo en forma cuadrática, lo que da lugar a que altitudes superiores a $1300 \mathrm{~m}$ la tendencia creciente en la estimación del $p F$ se invierta y disminuya (Fig.6). El modelo a $p F 4,2$ incluye la curvatura del perfil de máxima pendiente $[\mathrm{CuP}]$, haciéndolo de forma no lineal, con un aumento del contenido de humedad en las áreas de curvatura convexa y disminuyendo en las áreas cóncavas. Esta influencia en los modelos de los valores topográficos coinciden con los resultados obtenidos en otros trabajos (Poggio et al., 2010), según los cuales, los parámetros derivados del DTM demostraron su utilidad como información auxiliar en la predicción de los valores de capacidad de agua disponible en múltiples extensiones espaciales.

En el bloque C, solo las variables [Pr] y [Tmp] participan en el modelo $p F 4,2$. Su efecto es similar y actúan del mismo modo, aumentando el contenido de humedad a mayor temperatura y precipitación. Respecto a la radiación, [RaDi] y [RaDr] muestran un comportamiento similar en los dos modelos de $p F$ : en el caso de la radiación difusa el efecto es decreciente conforme aumentan los valores radiación, mientras que para la radiación directa el efecto 
se amplía. La variable [RaDu] solo aparece en el modelo $p F$ 2,5, actuando con un efecto no lineal produciendo un incremento del contenido de humedad a $p F$ 2,5 a medida que aumentan las horas de insolación.

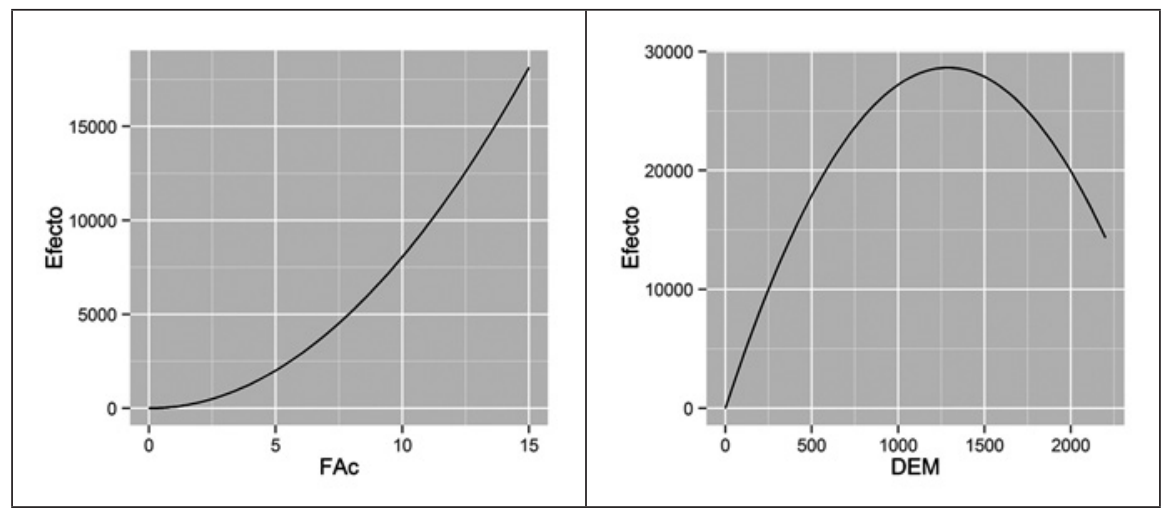

Finalmente, el bloque RS solo aparece presente con $\left[\mathrm{IOI}_{\mathrm{feb}}\right]$ para el modelo de $p F 2,5 \mathrm{y}$ $\left[\mathrm{NDVI}_{\mathrm{jul}}\right]$ para los dos modelos de $p F$. Respecto al índice mineralógico, los efectos se traducen en un descenso de los valores del $p F$ 2,5 conforme aumentan los niveles de alteración mineralógica para los elementos detectados en estos niveles del espectro electromagnético. Para $\left[\mathrm{NDVI}_{\mathrm{jul}}\right]$, los efectos son similares para los dos modelos, observándose un aumento de los niveles estimados de $p F$ a medida que aumentan los valores del índice de vegetación (Figuras 7 y 8).Esta información resulta bastante consistente, generando poco sesgo en el tipo de ecosistema asociado a la actividad vegetal, que es lo que realmente representa dicha variable, mostrando una conexión entre desarrollo vegetativo y capacidad de agua disponible en el suelo (Todoroff et al., 2010).

La interpretación de las variables cualitativas y sus efectos en los $p F$ se muestran en la figura 9. La fase salina de los datos LUCDEME [SPLu], se presenta como una variable cualitativa que contiene una información booleana, en la que la asignación del valor 1 o 0 a la porción delimitada por cada polígono expresa la presencia o ausencia de salinidad. Por su parte, siguiendo los criterios metodológicos expresados anteriormente, los efectos de las categorías de la variable Litología [LM] han sido evaluados con referencia a la clase 'Aluvial'. Las superficies en Fase Salina [SPLu] presentan un aumento de los valores para los dos modelos de $p F$, siendo la única variable cualitativa que afecta al $p F 2,5$. En el modelo $p F 4,2$ la variable $[\mathrm{LM}]$ actúa casi en la totalidad de sus categorías con un efecto negativo en el $p F$ respecto al nivel de referencia 'Aluvial', en tanto que las clases 'Coluvios' y 'Metamórfico Silíceo' alcanzan unos valores más extremos. Solo la categoría 'Dolomías', y de manera muy débil, muestra un efecto positivo. 


\section{Figuras 7 y 8}

REPRESENTACIÓN DEL ÍNDICE DE VEGETACIÓN (NDVI JUL) EN LOS MODELOS DE ESTIMACIÓN DEL pF 2,5 (IZQUIERDA) Y DEL $p F$ 4,2 (DERECHA), EN RELACIÓN CON EL EFECTO PRODUCIDO EN LA PF Y LOS VALORES PREDICTORES DEL MODELO

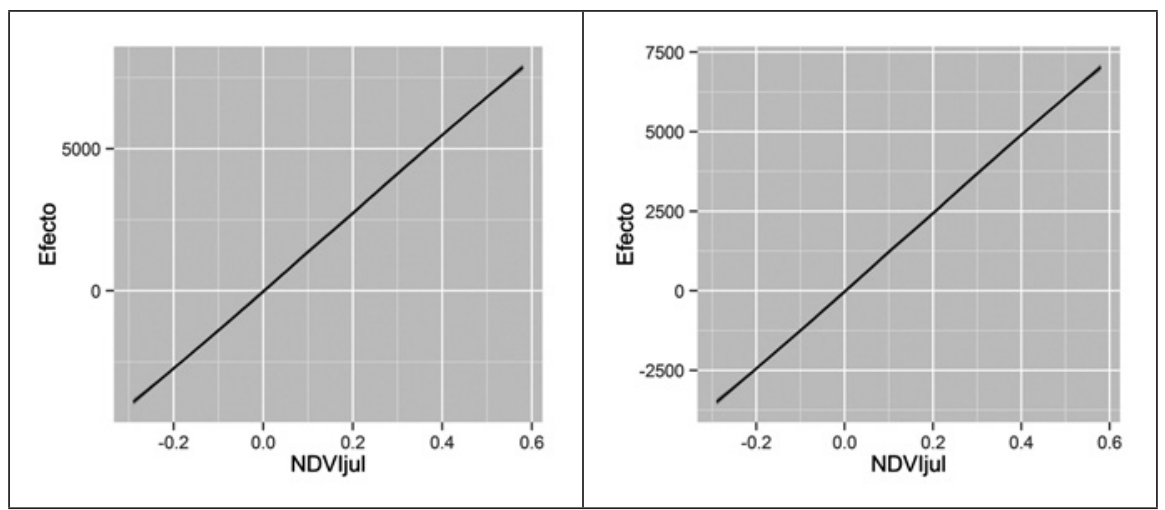

Figura 9

HISTOGRAMA DE LOS VALORES DE RECLASIFICACIÓN DE LAS PRINCIPALES CLASES DE LA VARIABLE LITOLOGIAA (LM), EXPRESADOS POR EL EFECTO PRODUCIDO EN EL PF Y LOS VALORES PREDICTORES DEL MODELO

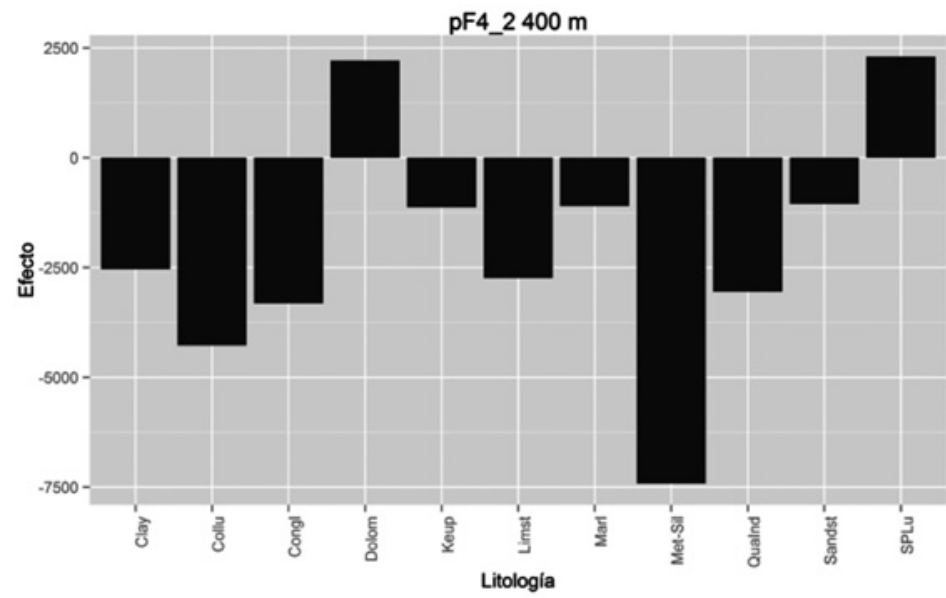

\section{CONCLUSIONES}

Los modelos de contenido de humedad del suelo a distintos $p F$ difieren estructuralmente, sugiriendo que los procesos ambientales que determinan la capacidad de retención de humedad del suelo a distintos $p F$ son, al menos parcialmente, diferentes en uno u otro caso. Estos resultados muestran una menor complejidad del modelo $p F 2,5$, respecto al modelo de $p F 4,2$. 
Para el modelo de $p F 4,2$, las variables que están mejor representadas se encuentran en los bloques [TG] y [C].Los efectos descritos por DTM, CuP y FAc a nivel del Punto de Marchitez están asociados a un incremento de los $p F$ en zonas de elevada altitud (hasta los 1500 $\mathrm{m})$, en superficies cóncavas y en áreas de drenaje de mayor entidad.

También es significativo el efecto del $\mathrm{NDVI}_{\mathrm{jul}}$, que participa en los dos modelos de $p F$, mostrando un aumento de los valores de $p F$ en zonas de mayor vegetación, causado por la relación entre materia orgánica y vegetación con la capacidad de retención de humedad del suelo.

Por último, para nuestros resultados, se puede afirmar que la información aportada por teledetección ha permitido mejorar los modelos, por lo que el uso de datos obtenidos por sensores remotos puede ser recomendable en combinación con el resto de variables ambientales.

\section{REFERENCIAS BIBLIOGRÁFICAS}

AGUSTÍ, M. (2003). Citricultura. Madrid, Ediciones Mundi-Prensa.

AKAIKE, H. (1974). «A new look at the statistical model identification». IEEE Transactions on Automatic Control, $\mathrm{n}^{\circ} 19:$ : 716-723.

ALONSO-SARRÍA, F. (2007). Atlas Global de la Región de Murcia. La Verdad- CMM S.A. Murcia. Disponible en: http://www.atlasdemurcia.com/index.php/secciones/1/el-clima/3/

ÁLVAREZ ROGEL, J.; ORTIZ SILLA, R. y ALCARAZ ARIZA, F. (2001). «Edaphic characterization and soil ionic composition influencing plant zonation in a semiarid Mediterranean salt marsh».Geoderma, nº99: 81-98.

BANDARA, R., WALKER, J.P. y RUDIGER, C. (2013). «Towards soil property retrieval from space: Aone-dimensional twin-experiment». Journal of Hydrology 497: 198-207.

BEN-DOR, E. (2002). «Quantitative remote sensing of soil properties».Advances in Agronomy, $\mathrm{n}^{\circ} 75,173-243$.

BEN-DOR, E.; CHABRILLAT, S.; DEMATTÊ, J.A.M.; TAYLOR, G.R.; HILL, J.; WHITING, M.L. y SOMMER,S.(2009). «Using Imaging Spectroscopy to study soil properties». Remote Sensing of Environment, $\mathrm{n}^{\circ} 113$ : 38-55.

BOIX-FAYOS, C.; MARTÍNEZ-MENA, M.; CALVO-CASES, A.; CASTILLO, V. y ALBALADEJO, J.(2005). «Concise review of interrill erosion studies in SE Spain (Alicante and Murcia): Erosion rates and progress of knowledge from the 1980〉s». Land Degradation andDevelopment, $\mathrm{n}^{\circ} 16: 517-528$.

BRUS, D.J. y DE GRUIJTER, J.J.(1997). «Random sampling or geostatistical modelling. Choosing between design-based and model-based sampling strategies for soil». Geoderma, ${ }^{\circ} 80,1-59$.

BURROUGH, P.A.; VAN GAANS, P.F.M. y HOOTSMANS, R. (1997).«Continuous classification in soil survey: Spatial correlation, confusion and boundaries». Geoderma, $\mathrm{n}^{\circ} 77$ : $115-135$.

BURROUGH, P.A. y MCDONNELL, R.A. (1998). Principles of geographical information systems.Oxford University Press, Oxford.

BUCCI, S.J., SCHOLZ, F.G., GOLDSTEIN, G., MEINZER, F.C. y ARCE, M.E. (2009). «Soil wateravailability and rooting depth as determinants of hydraulic architecture of Patagonian woody species». Oecologia 160 (4): 631-641. 
BOU KHEIR, R.; GREVE, M.H.; BØCHER, P.K.; GREVE, M.B.; LARSEN, R. y MCCLOY, K.(2010). «Predictive mapping of soil organic carbon in wet cultivated lands usingclassification-tree based models: The case study of Denmark». Journal of Environmental Management, $\mathrm{n}^{\circ}$ 91, 1150-1160.

CAPEL-MOLINA, J.J. (2000). El clima de la Península Ibérica.Ariel Geografía, Barcelona.

CHEN, F.; KISSEL, D.E.; WEST, L.T. y ADKINS, W. (2000). «Field-scale mapping of surface soil organic carbon using remotely sensed imagery». Soil Science Society of America Journal, 64: 746-753.

CONESA GARCÍA, C., y ALONSO SARRIA, F. (2006). El Clima de la Región de Murcia. En ConesaGarcía, C. (Ed.), El Medio Físico de la Región de Murcia. Servicio de Publicaciones, Universidad de Murcia. Murcia, 3: 95-127.

CONFEDERACIÓN HIDROGRÁFICA DEL SEGURA (CHS), Ministerio de Agricultura, Alimentación y Medio Ambiente. 2013. Disponible en: http://www.chsegura.es/chs/ cuenca/-resumendedatosbasicos/marcoadministrativo/

CROSTA, A.P.; DE SOUZA FILHO, C.R.; AZEVEDO, F. y BRODIE, C. (2003). «Targeting key alteration minerals in epithermal deposits in Patagonia, Argentina, using ASTER imagery and principal component analysis». International Journal of Remote Sensing, n $24: 4233-4240$.

DOBOS, E. y HENGL, T. (2009). Soil mapping applications. In: T. Hengl and H.I. Reuter, editors, Geomorphometry, concepts, software and applications. Elsevier, New York, 461-479.

DUNKERLEY, D.L. (2002). «Infiltration rates and soil moisture in a grovedmulga communitynear Alice Springs, arid central Australia: Evidence for complex internal rainwaterredistribution in a runoff-runon landscape». Journal of Arid Environments 51 (2):199-219.

DURY, S.A. (1987). Image Interpretation in Geology. Chapman \& Hall. London.

ESRI 2013. ArcGIS Desktop: Release 10. Redlands, CA: Environmental Systems Research Institute.

FAO (1974). Soil Map of the World.Vol I.

FOX, G.A. y SABBAGH, G.J. (2002). «Estimation of soilorganic matter from red and nearinfrared remotely sensed data using soil line Euclidean techniques».Soil Science Society of America Journal, 66: 1922-1929.

FU, P.y P. M. RICH. (2000). The Solar Analyst 1.0 Manual. Helios Environmental

GARCIA-ESTRINGANA, P., ALONSO-BLAZQUEZ, N. y ALEGRE, J. (2010). «Water storage capacity,stemflow and water funneling in Mediterranean shrubs». Journal of Hydrology 389(3-4) : 363-372.

GESSLER, P.E.; MOORE, I.D.; MCKENZIE, N.J. y RYAN, P.J. (1995).«Soil-landscape modelling and spatial prediction of soil attributes». Int. J. Geographical Information Systems, $\mathrm{n}^{\circ}$ 4, 421-432.

GREVE, M.H.; KHEIR, R.B.; GREVE, M.B. y BØCHER, P.K. (2012). «Quantifying the ability of environmental parameters to predict soil texture fractions using regression-tree model with GIS and LIDAR data: The case study of Denmark». Ecol. Indic. $\mathrm{n}^{\circ}$ 18, 1-10.

HERWITZ, S.R. (1986). «Infiltration-excess caused by stemflow in a cyclone prone tropical rainforest». Earth Surface Processes \& Landforms, 11 (4): 401-412.

ICONA. Proyecto LUCDEME (Lucha contra la Desertificacion del Mediterraneo) (1986). Mapa de suelos, escala 1:100.000. ICONA - Ministerio de Agricultura, Pesca y Alimentación. 
INSTITUTO NACIONAL DE ESTADÍSTICA (INE). Ministerio de Economía y Competitividad. 2013. Disponible en: http://www.ine.es/inebmenu/mnu_entornofis.htm\#1.

KIDNER, D.; DOREY, M. y SMITH D.(1999). «What>s the point? Interpolation and extrapolation with a regular grid DTM». Proceedings of the 4th International Conference on GeoComputation.

KLUTE, A. (1986). Water retention: Laboratory methods, in A.Klute, ed. Methods of Soil Analysis. Part 1: Physical and Mineralogical Methods. AgranomyMonograpfh 9, ASA, Madison, WI, pp. 635-662.

LAGACHERIE, P.; BAILLY, J.S.; MONESTIEZ, P. y GOMEZ, C. (2012). «Using scattered hyperspectral imagery data to map the soil properties of a region». European Journal of SoilScience, $\mathrm{n}^{\circ}$ 63: 110-119.

LBI, (2013). Laboratorio de Biogeografía Informática. Museo Nacional de Ciencias Naturales. CSIC. Disponible en: http://www.mncn.csic.es/Menu/Investigacin/Serviciodeapoyoalainves-tigacin/LabBiogeografaInformtica/seccion=1258\&idioma=es_ES.do

LIEß, M.; GLASER, B. y HUWE, B. (2012). «Uncertainty in the spatial prediction of soil texture Comparison of regression tree and Random Forest models». Geoderma, $\mathrm{n}^{\circ} 170$, 70- 79.

LOUSSERT, R. (1992). Los Agrios. Madrid, Ediciones Mundi-Prensa.

MALIK, R.S., BUTTER, B.S., ANALAUF, R. y RICHTER, J., (1987). «Water penetration into soils with different textures and initial soil contents». Soil Science, 144(6): 389-393.

MANFREDA, S., SCANLON, T.M., CAYLOR, K.K. (2010). On the importance of accurate depictionof infiltration processes on modelled soil moisture and vegetation water stress. Ecohydrology 3 (2): 155-165.

MCBRATNEY, A.B.; WEBSTER, R. y BURGESS, T.M. (1981).«The design of optimal samplingschemes for local estimation and mapping of of regionalized variables-I. Theoryand method».Computers and Geosciences, $\mathrm{n}^{\circ}$ 7: 331-334.

MCBRATNEY, A.B.; MENDONÇA SANTOS, M.L. y MINASNY, B. (2003). On digital soil mapping. Geoderma, n ${ }^{\circ}$ 117: 3-52.

MCKENZIE, N.J. y RYAN, P.J. (1999). Spatial prediction of soil properties usingenvironmental correlation. Geoderma, $\mathrm{n}^{\circ}$ 89: 67-94.

MINASNY, B., y MCBRATNEY, A.B. (2008). «Regression rules as a tool for predicting soil properties from infrared reflectance spectroscopy». Chemom. Intell. Lab. Syst., n ${ }^{\circ}$ 94: 72-74.

MINISTERIO DE MEDIO AMBIENTE (MMA) (2002-2012). Inventario Nacional de Erosión de Suelos. Disponible en: http://www.magrama.gob.es/es/biodiversidad/temas/ inventariosnacio-nales/inventarionacional-de-erosion-de-suelos/.

MOORE, I.D.; GRAYSON, R.B.y LANDSON, A.R. (1991).«Digital Terrain Modelling: A Review of Hydrological, Geomorphological, and Biological Applications».Hydrological Processes, $\mathrm{n}^{\circ}$ 5, 3-30.

NASA - METI. (2013). ASTER GDEM.The Ministry of Economy, Trade and Industry of Japan (METI) and the NationalAeronautics and Space Administration (NASA). Disponible en: http://gdem.ersdac.jspacesystems.or.jp/.

PEREZ-CUTILLAS, P. Modelización de propiedades físicas del suelo a escala regional. Casos de estudio en el Sureste Ibérico. Tesis Doctoral. Universidad de Murcia. 2013. Disponible en: http://hdl.handle.net/10201/37810 
POGGIO, L., GIMONA, A., BROWN, I. y CASTELLAZZI, M. (2010). «Soil available water capacityinterpolation and spatial uncertainty modelling at multiple geographical extents». Geoderma 160 (2): 175-188.

POGGIO, L.; GIMONA, A. y BREWER, M.J. (2013). «Regional scale mapping of soil properties and their uncertainty with a large number of satellite-derived covariates».Geoderma, $\mathrm{n}^{\circ}$ 209-210: 1-14.

RAWLS, W.; COSH, M.; JACKSON, T. y NEMES, A. (2004). «Use of remotely sensed soil moisture to determine soil hydraulic properties». International Geoscience and Remote Sensing Symposium (IGARSS), $\mathrm{n}^{\circ}$ 4, 2791-2794.

RICHARDS, L.A. (1947). Pressure membrane apparatus: construction and use. Agric. Eng., 28: 451-454.

SABINS JR, F.F. (1981). Geological applications and training in remote sensing. CORSE 81: the 1981 conference on remote sensing education, Lafayette, IN: 77-82.

R CORE TEAM (2013). R: A language and environment for statistical computing. R Foundation for Statistical Computing, Vienna, Austria.URL http://www.R-project.org/.

RICH, P. M.; DUBAYAH,R.; HETRICK,W.A.y SAVING, S.C. (1994). «Using Viewshed Models to Calculate Intercepted Solar Radiation: Applications in Ecology». American Society for Photogrammetry and Remote Sensing Technical Papers, 524-529.

SCHUH, W.M. y CLINE, R.L. (1990). «Effect of soil properties on unsaturated hydraulic conductivity pore interaction factors». Soil Science Society of America Journal. 54(6): 1509-1519.

SCHULTEN, H.R. y SCHNITZER, M. (1997). «Chemical model structures for soil organic matter and soils».Soil Science, $\mathrm{n}^{\circ} 162,115-130$.

SCULL, P.; FRANKLIN, J.; CHADWICK, O.A. y MCARTHUR, D. (2002). «Predictive soil mapping: Areview». Progress in Physical Geography, n 27, 171-197.

TARBOTON, D. G.; BRAS, R. L. y RODRIGUEZ-ITURBE, I. (1991). «On the Extraction of Channel Networks from Digital Elevation Data».Hydrological Processes, $\mathrm{n}^{\circ}$ 5, 81-100.

THOMPSON, J.A.; BELL, J.C. y BUTLER, C.A. (2001). «Digital elevation model resolution: Effects on terrain attribute calculation and quantitative soil-landscape modeling». Geoderma, $\mathrm{n}^{\circ}$ 100: 67-89.

TODOROFF, P., DE ROBILLARD, F. y LAURENT, J.-B. (2010). «Document Interconnection of a crop growth model with remote sensing data to estimate the total available water capacity of soils».International Geoscience and Remote Sensing Symposium (IGARSS). Article 5653790, Pages 1641-1644

TOWNSHEND, J.R.G.; GOFF, T.E. y TUCKER, C.J. (1985).«Multitemporal dimensionality of images of Normalized Difference Vegetation Index at continental scales». IEEE Transactions on Geoscience and Remote sensing, $\mathrm{n}^{\circ} 23: 888-895$.

TWARAKAVI, N.K.C, ŠIMUNEK, J. y SCHAAP, M.G. (2010). «Can texture-based classificationoptimally classify soils with respect to soil hydraulics?». Water Resources Research 46 (1), art. no. W01501

UNO, Y., PRASHER, S.O., PATEL, R.M., STRACHAN, I.B. y PATTEY, E. (2005). «Development of field-scale soil organic matter content estimation models in Eastern Canada using airborne hyperspectral imagery». Canadian Biosystems Engineering/Le génie des biosystèmes au Canada, 47: 9-14. 
VAN GENUCHTEN, M.TH. (1980). «Closed-form equation for predicting the hydraulicconductivity of unsaturated soil». Soil Sci. Soc. Am, 44: 1147-1152.

VISCARRA-ROSSEL, R.A.; WALVOORT, D.J.J.; MCBRATNEY, A.B.; JANIK, L.J. y SKJEMSTAD, J.O. (2006). «Visible, near infrared, mid infrared or combined diffuse reflectance spectroscopyfor simultaneous assessment of various soil properties». Geoderma, $\mathrm{n}^{\circ} 131: 59-75$.

VRIELING, A. (2006). «Satellite remote sensing for water erosion assessment: A review». Catena, $\mathrm{n}^{\circ} 65,2-18$.

WANG, K., ZHANG, C., LI, W. (2013). «Predictive mapping of soil total nitrogen at a regionalscale: A comparison between geographically weighted regression and cokriging». Applied Geography, 42: 73-85.

WESTERN, A.W.; ZHOU, S.L.; GRAYSON, R.B.; MCMAHON, T.A.; BLÖSCHL, G. y WILSON, D.J. (2004). «Spatial correlation of soil moisture in small catchments and its relationship todominant spatial hydrological processes ».Journal of Hydrology, $\mathrm{n}^{\circ}$ 286,113-134.

WHEELER, S.J., SHARMA, R.S., BUISSON, M.S.R. (2003). «Coupling of hydraulic hysteresis andstress-strain behaviour in unsaturated soils». Geotechnique, 53(1): 41-54.

WOSTEN, J.H.M. y VAN GENUCHTEN, M.T. (1988). «Using texture and other soil propertiesto predict the unsaturated soil hydraulic functions». Soil Science Society of America Journal, 52 (6): 1762-1770.

ZEVERBERGEN, L.W. y THORNE, C.R.(1987). «Quantitative Analysis of Land Surface Topography». Earth Surface Processes and Landforms, $\mathrm{n}^{\circ}$ 12: 47-56. 
